\title{
Nonintuitive interplay between confinement and dimensionality for angle-resolved first passage statistics
}

\author{
Charles Antoine (1)* and Julian Talbot ${ }^{\dagger}{ }^{\dagger}$ \\ Laboratoire de Physique Théorique de la Matière Condensée, UPMC, CNRS, UMR No. 7600, \\ Sorbonne Universités, 4 Place Jussieu, 75252 Paris Cedex 05, France
}

(Received 10 July 2019; revised manuscript received 30 October 2019; published 21 January 2020)

\begin{abstract}
First passage processes often seem to defy common sense. For example, consider a particle diffusing toward a target in a spherically symmetric confined system. One might naively expect that the hitting angle distribution as the confining boundary recedes to infinity would be the same as for an unbounded system. Here we show that this may or may not be true depending on the spatial dimension. In two dimensions, the limiting hitting distribution corresponds to that of the unbounded system, while in three (or more) dimensions the two differ by a nonintuitive additive constant, even when one accounts for the well-known finite probability that the particle never reaches the target. Confinement has other subtle effects on the features of the angular hitting distribution. For example, depending on the dimension and the starting distance from the target, the boundary may differentially favor arrival opposite to the entry point and lead to a maximum in the three-dimensional variance of the hitting angle statistics.
\end{abstract}

DOI: 10.1103/PhysRevResearch.2.012019

Imagine the following scenarios. (i) A defenseless animal is randomly searching for a source of water in a new, entirely closed territory, e.g., a national park, where a small lake is hidden [1-6]. The shoreline of this water point is particularly dangerous since well-camouflaged predators are on the prowl, except at one small location at the rear of the lake where it is safe. Will the wandering animal be able to ease its thirst without being devoured? (ii) Therapeutic molecules are diffusing in a cell and to be effective they must reach a specific part of the nucleus [7-11]. In both situations we are interested in the probability for a diffusive agent to hit a specific point on a target in a confined space and, more subtly, does the degree of confinement play any significant role in this random search? As we will see in the rest of this Rapid Communication, the answer may be dramatically different depending on whether the diffusive search is in two (the animal) or three (the drug molecule) dimensions.

The questions naturally fall within the scope of first passage processes [12-15] that find wide application. In ecology, examples include animal foraging [16-20], prey location [21], mating encounters [22], and the effect of physical barriers on animal movement [23] and predator-prey dynamics [4-6]. Diffusion limited reactions like the binding time of a polymer to a surface receptor [24], the escape time from one phenotypic state to another during breast cancer growth [25] or the time needed for proteins to search for particular DNA locations [26-28] can also be formulated in terms of first

\footnotetext{
*antoine@1ptmc.jussieu.fr

${ }^{\dagger}$ talbot@lptmc.jussieu.fr
}

Published by the American Physical Society under the terms of the Creative Commons Attribution 4.0 International license. Further distribution of this work must maintain attribution to the author(s) and the published article's title, journal citation, and DOI. passage times. Diffusion of cold atoms in optical lattices [29], oxygen transport in muscle tissue [30], and self-assembly of macromolecules [31] are yet further examples.

First passage processes in confined geometries [32-37] are of particular interest because of their relevance for problems in biophysics. The boundary may be totally or partially reflecting [38], with narrow [39,40] or large escape zones and/or absorbing areas which play the role of the target. A recent application of such confined processes concerns intracellular targeting for therapeutic intervention on, e.g., autoimmune, oncogenic, and degenerative diseases [7-11,41-44].

The eventual hitting location of a diffusing particle on an object is controlled by its harmonic measure [45]. Physically, this corresponds to the gradient of the electrostatic potential at the object's boundary. The harmonic measure of fractal structures formed by diffusion limited aggregation has been well studied [46-49]. For regular, nonfractal, targets, however, there are very few studies on the effect of confinement on the hitting position $[48,50]$ and recent studies of first passage processes [51-55] focus on the first passage time rather than the hitting location.

In the following we present analytic solutions for the hitting angle distribution on a uniform circular [two-dimensional (2D)] or spherical (3D) target contained within a spherically symmetric reflecting wall. The diffusing particle is absorbed, and its trajectory ends, the first time it touches any point on the target. We suppose that there is a preferred location on the target, and for the sake of argument we assume that it is somewhere behind the target relative to the starting position. Our model should be contrasted with narrow escape problems $[40,56,57]$ in which the trajectory of the diffusing particle ends only when it exits through one or more small windows.

Two dimensions. The eventual hitting angle distribution in an unbounded system may be found by using a mapping to the equivalent electrostatic system [12] (see Fig. 1). More precisely, the hitting probability at a boundary point 


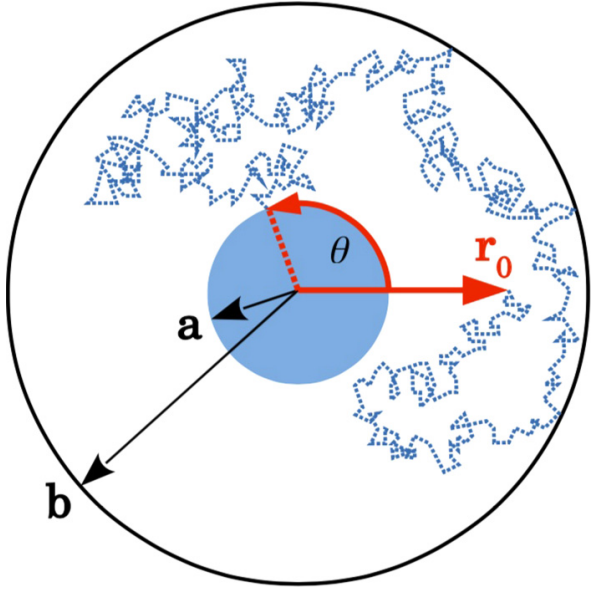

FIG. 1. The two-dimensional system consists of a Brownian particle diffusing in an annulus formed from an inner absorbing target of radius $a$ and an outer reflecting wall of radius $b$. The particle starts its diffusive trajectory at a distance $r_{0}$ from the center and terminates when it touches the target for the first time. We are interested in the distribution of the angle of the point of first contact on the target, $\theta$.

corresponds to the electric field at the same location when a charge of appropriate magnitude is placed at a distance $r_{0}$ and the boundary surface is grounded. The electric field can be found using the method of images and the result for the eventual hitting probability is

$$
f_{2 \mathrm{D}}^{*}(\theta)=\frac{1}{2 \pi} \frac{1-a^{2} / r_{0}^{2}}{1-\left(2 a / r_{0}\right) \cos \theta+a^{2} / r_{0}^{2}},
$$

where the asterisk indicates the absence of a confining boundary (see, e.g., [12]). The distribution is normalized $\int_{-\pi}^{\pi} f_{2 \mathrm{D}}^{*}(\theta) d \theta=1$, as the particle is certain to eventually hit the target, and has maximum and minimum values at $\theta=0$ and $\theta=\pi$, respectively. Few published studies have attempted to extend these results to confined systems, but see $[48,50]$, with the former considering confinement by flat planes.

More generally, it can be shown [58] that $f(r, \theta)$ satisfies the harmonic equation

$$
\frac{1}{r_{0}} \frac{\partial}{\partial r_{0}}\left(r_{0} \frac{\partial f_{2 \mathrm{D}}}{\partial r_{0}}\right)+\frac{1}{r_{0}^{2}} \frac{\partial^{2} f_{2 \mathrm{D}}}{\partial \theta^{2}}=0,
$$

with boundary conditions $f(a, \theta)=\delta(\theta)$ and $\partial f\left(r_{0}, \theta\right) /$ $\left.\partial r_{0}\right|_{r_{0}=b}=0$. This leads to the solution

$$
f_{2 \mathrm{D}}\left(r_{0}, \theta\right)=\frac{1}{2 \pi}\left[1+2 \sum_{m=1}^{\infty} c_{m} \cos (m \theta)\right],
$$

where

$$
c_{m}=\frac{r_{0}^{2 m}+b^{2 m}}{a^{2 m}+b^{2 m}}\left(\frac{a}{r_{0}}\right)^{m}
$$

is a decreasing function of $b$ and $r_{0}$.

This distribution is clearly normalized, $\int_{-\pi}^{\pi} d \theta f_{2 \mathrm{D}}(\theta)=1$, which is consistent with the certainty of (eventually) hitting the target. Some results are shown in Fig. 2. One observes that the more confined the system, the sharper the distribution: The

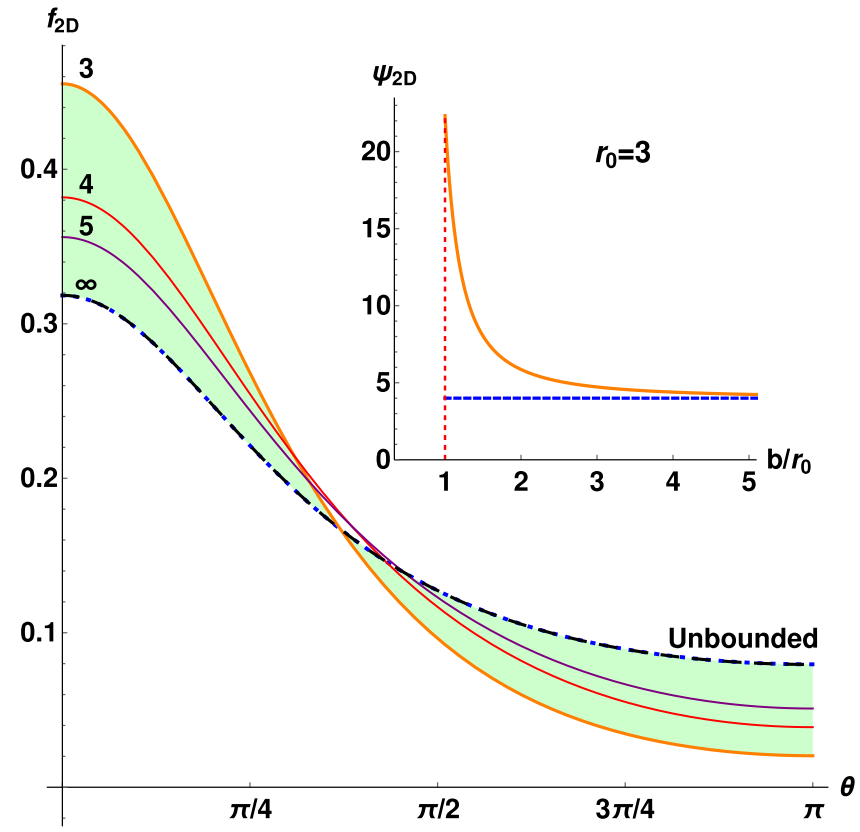

FIG. 2. Eventual hitting angle distribution in a 2D system (3) for a starting distance $r_{0}=3$ and different confinements $b=3,4,5$ ( $a=$ 1). The limiting distribution $(b=\infty)$, shown as the dashed curve, is the same as the unbounded one (1). The inset shows the ratio of the maximum to the minimum hitting probability $\psi\left(b, r_{0}\right)$.

particle is more likely to hit at $\theta=0$ and less likely to reach the rear of the target at $\theta=\pi$ than in an unconfined system.

One can show that for large $b$ the bounded and unbounded [Eq. (1)] hitting angle distributions are related as

$f_{2 \mathrm{D}}(\theta)=f_{2 \mathrm{D}}^{*}(\theta)+\frac{a}{r_{0}}\left[\left(\frac{r_{0}}{a}\right)^{2}-1\right]\left(\frac{a}{b}\right)^{2} \cos \theta+O\left((a / b)^{4}\right)$,

showing that they are equal in the limit of an infinitely distant boundary. Moreover, convergence to this limit is rapid unless the diffusing particle starts close to the external confining boundary $r_{0} \approx b$.

The mean and mean square of the cosine of the hitting angle are given by

$$
\begin{gathered}
\langle\cos \theta\rangle=c_{1}=\frac{r_{0}^{2}+b^{2}}{a^{2}+b^{2}}\left(\frac{a}{r_{0}}\right), \\
\left\langle\cos ^{2} \theta\right\rangle=\left(1+c_{2}\right) / 2=\frac{1}{2}\left[1+\frac{r_{0}^{4}+b^{4}}{a^{4}+b^{4}}\left(\frac{a}{r_{0}}\right)^{2}\right]
\end{gathered}
$$

and these decrease monotonically with increasing $b$ for fixed initial position $r_{0}$ and with increasing $r_{0}$ for fixed $b$. For a confined 2D system ( $b$ finite), the mean of the cosine of the hitting angle is always larger than for the unbounded system. The variance increases monotonically with increasing $r_{0}$ for fixed $b$ and with increasing $b$ for fixed $r_{0}$ and is always smaller than for the unbounded system [58].

Further insight into the effect of confinement on the hitting angle distribution is provided by the ratio of the maximum to the minimum probabilities $\psi\left(r_{0}\right)=f\left(r_{0}, 0\right) / f\left(r_{0}, \pi\right)$ [12]. In two dimensions confinement always increases this ratio compared to an unconfined system with the same starting distance 
$\psi\left(r_{0}\right)>\psi^{*}\left(r_{0}\right)$, i.e., confinement preferentially favors hitting the front compared with the back of the disk, decreasing both the mean angle and the probability of hitting the preferred region of the target (see Fig. 2).

To understand this behavior, we recall that in two dimensions all particles eventually hit the target. Those that take a long time to arrive are more likely to have a random orientation with respect to the starting position, thus enhancing the probability to arrive at the rear of the target. When a barrier is present, it tends to suppress this effect by preventing the particles from drifting too far away from the target. Thus they retain some correlation with the starting position and are more likely to hit the front of the target.

Three dimensions. The eventual hitting probability for an unbounded system

$$
f_{3 \mathrm{D}}^{*}(\theta)=\frac{a}{2 r_{0}} \frac{1-a^{2} / r_{0}^{2}}{\left[1-\left(2 a / r_{0}\right) \cos \theta+a^{2} / r_{0}^{2}\right]^{3 / 2}}
$$

is not normalized $\int_{0}^{\pi} d \theta \sin \theta f_{3 \mathrm{D}}^{*}(\theta)=a / r_{0}<1$, as in three dimensions there is a finite probability that the particle never reaches the target. For the bounded system consisting of a spherical target contained within a spherical reflecting boundary of radius $b$ we obtain, using a similar approach as for the 2D system,

$$
f_{3 \mathrm{D}}(\theta)=\frac{1}{2}+\sum_{m=1}^{\infty}\left(m+\frac{1}{2}\right) \alpha_{m} P_{m}(\cos \theta),
$$

where $P_{m}(x)$ is the Legendre polynomial and

$$
\alpha_{m}=\frac{(m+1) r_{0}^{2 m+1}+m b^{2 m+1}}{(m+1) a^{2 m+1}+m b^{2 m+1}}\left(\frac{a}{r_{0}}\right)^{m+1} .
$$

Clearly, $\int_{0}^{\pi} d \theta \sin \theta f_{3 \mathrm{D}}(\theta)=1$, i.e., the particle is certain to hit the target as a result of the confining wall.

Naively, we might expect that the distribution of the bounded system as the reflecting wall recedes to infinity $f_{3 \mathrm{D}}^{\infty}\left(r_{0}, \theta\right)$ would just be the unbounded distribution normalized $\left(r_{0} / a\right) f_{3 \mathrm{D}}^{*}\left(r_{0}, \theta\right)$. However, this significantly underestimates the probability of hitting the backside of the target in the confined system. In fact, one can show that

$$
\begin{aligned}
f_{3 \mathrm{D}}(\theta)= & f_{3 \mathrm{D}}^{*}\left(r_{0}, \theta\right)+\frac{1}{2}\left(1-\frac{a}{r_{0}}\right) \\
& +3\left(\frac{a}{r_{0}}\right)^{2}\left[\left(\frac{r_{0}}{a}\right)^{3}-1\right]\left(\frac{a}{b}\right)^{3} \cos \theta+O\left(\left(\frac{a}{b}\right)^{5}\right),
\end{aligned}
$$

so in the limit of infinite $b$ the two differ by the additive constant $\frac{1}{2}\left(1-\frac{a}{r_{0}}\right)$. This can be understood in the following way. In the unbounded system, a fraction of particles $1-a / r_{0}$ never reaches the target. If a (distant) reflecting barrier is present, however, these particles are not lost and will eventually hit the target. Their hitting angle, however, is largely uncorrelated with the initial orientation and they therefore contribute a constant term to the bounded hitting angle distribution (the factor of $\frac{1}{2}$ results from the normalization factor $\left.\int_{0}^{\pi} d \theta \sin \theta\right)$. A graphical illustration of the difference is presented in Fig. 3. The first correction to the infinite limit occurs at order $(a / b)^{3}$ [Eq. (11)], explaining why the hitting

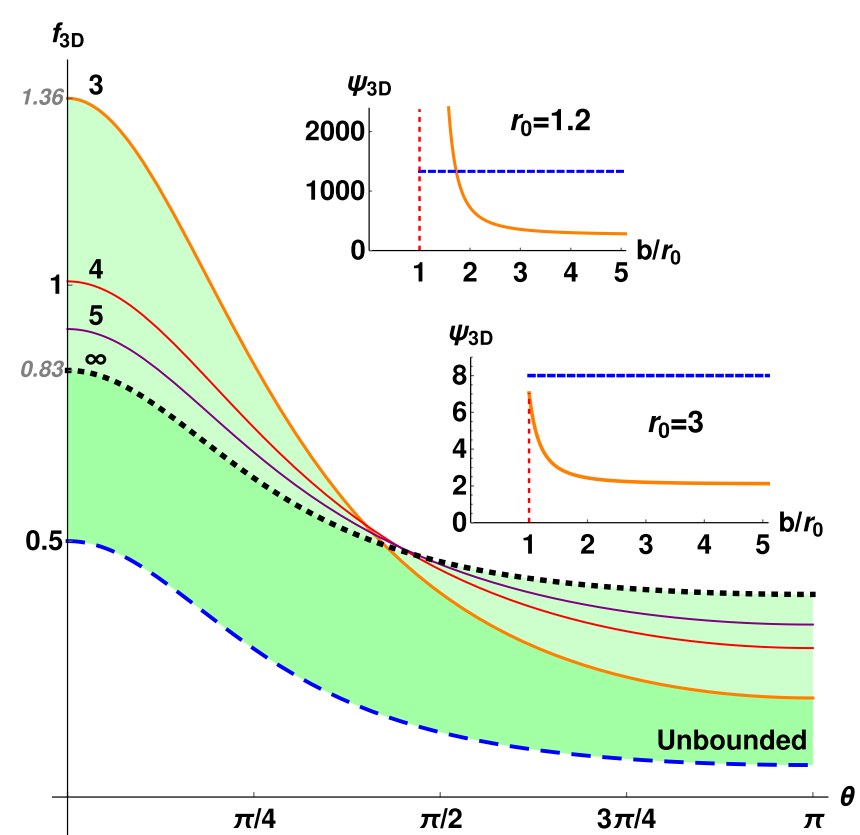

FIG. 3. Eventual hitting angle distribution in a $3 \mathrm{D}$ system for $r_{0}=3$ and $b=3,4,5(a=1)$. The dotted curve shows the limiting distribution (11) and the dashed curve shows the distribution in the unbounded system (8). The insets show $\psi\left(b, r_{0}\right)$ for two different values of the starting distance, $r_{0}=1.2<r_{0 c}$ and $r_{0}=3>r_{0 c}$, with $r_{0 c}$ defined in the text.

angle distribution converges rapidly to the infinite limit with increasing $b$. For example, for $r_{0}=2 a$, the confined distribution differs from the asymptotic one $f_{3 \mathrm{D}}^{*}\left(r_{0}, \theta\right)+\frac{1}{2}\left(1-\frac{a}{r_{0}}\right)$ by less than $10 \%$ for $b \geqslant 4 a$.

The behavior of the probability ratio $\psi\left(r_{0}\right)=$ $f_{3 \mathrm{D}}\left(r_{0}, 0\right) / f_{3 \mathrm{D}}\left(r_{0}, \pi\right)$ is more subtle than in two dimensions. If the starting point $r_{0}$ is farther than a specific distance $r_{0 c}=2.7944 a$, then $\psi\left(r_{0}\right)<\psi^{*}\left(r_{0}\right)$, i.e., any degree of confinement reduces the relative probability of hitting the front compared with the back, which is the opposite of the behavior observed in two dimensions. In three dimensions, only a fraction of the particles hits the target and those that do typically follow direct trajectories [37]. If a confining barrier is present, it channels some of the particles that would have escaped to the rear of the target, thus lowering $\psi$ compared with $\psi^{*}$.

If, on the other hand, $r_{0}<r_{0 c}$, we observe a crossover. For $b<r_{01}\left(r_{0}\right), \psi\left(r_{0}\right)>\psi^{*}\left(r_{0}\right)$, while if $b>r_{01}\left(r_{0}\right), \psi\left(r_{0}\right)<$ $\psi^{*}\left(r_{0}\right)$. In the former case, i.e., with a high degree of confinement, the barrier tends to reflect the particles directly back towards the target, thus enhancing the front to back hitting probability ratio $\psi$.

The first and second moments of $\cos \theta$ are

$$
\begin{gathered}
\langle\cos \theta\rangle=\frac{2 r_{0}^{3}+b^{3}}{2 a^{3}+b^{3}}\left(\frac{a}{r_{0}}\right)^{2}, \\
\left\langle\cos ^{2} \theta\right\rangle=\frac{1}{3}+\frac{2}{3}\left(\frac{3 r_{0}^{5}+2 b^{5}}{3 a^{5}+2 b^{5}}\right)\left(\frac{a}{r_{0}}\right)^{3},
\end{gathered}
$$

with asymptotic limiting values $\langle\cos \theta\rangle_{\infty}=\left(a / r_{0}\right)^{2}$ and $\left\langle\cos ^{2} \theta\right\rangle_{\infty}=\frac{1}{3}+\frac{2}{3}\left(a / r_{0}\right)^{3}$. These are different from the 


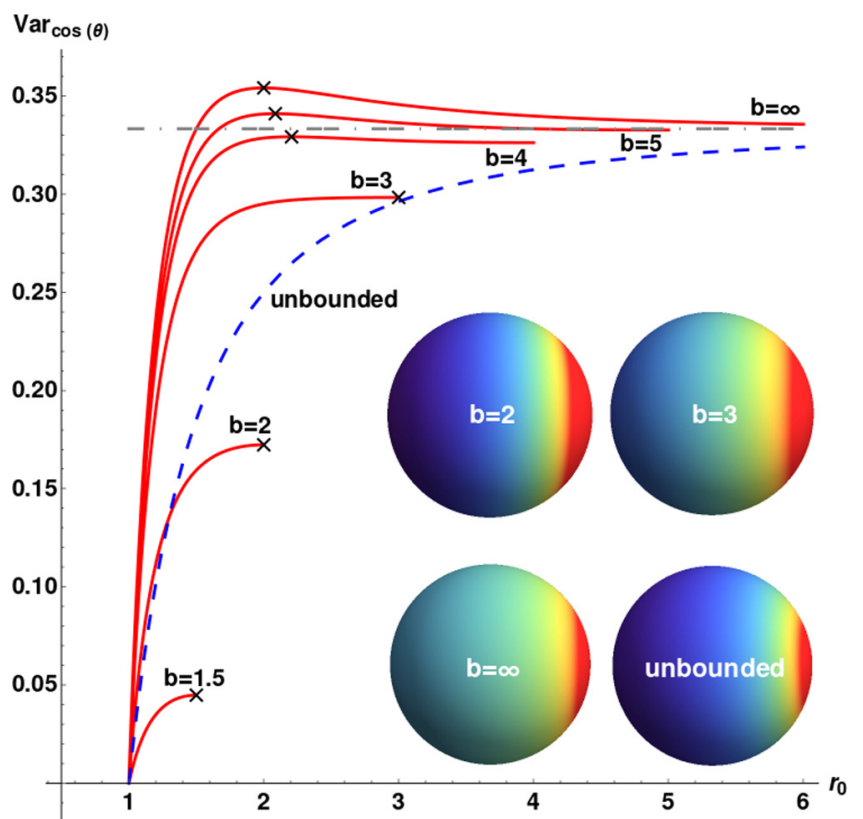

FIG. 4. Variance of the cosine of the hitting angle in the 3D system as a function of the starting position $r_{0}$ for different values of $b$ (solid red curves), compared with the unbounded system [1 $\left.\left(a / r_{0}\right)^{2}\right] / 3$ (dashed blue curve), and the uniform case (dot-dashed gray curve). The crosses indicate the position of the maxima. The inset shows the spherical target surface density plots for $r_{0}=2$ for different values of $b$ and for the unbounded system (bottom right).

unbounded values $\langle\cos \theta\rangle^{*}=a / r_{0}$ and $\left\langle\cos ^{2} \theta\right\rangle^{*}=\frac{1}{3}+$ $\frac{2}{3}\left(a / r_{0}\right)^{2}$. This is in stark contrast to the 2D system for which the corresponding quantities are the same, i.e., $\langle\cos \theta\rangle^{*}=$ $\lim _{b \rightarrow \infty}\langle\cos \theta\rangle$ and $\left\langle\cos ^{2} \theta\right\rangle^{*}=\lim _{b \rightarrow \infty}\left\langle\cos ^{2} \theta\right\rangle$.

In Fig. 4 we examine the variance of the cosine of the hitting angle as a function of the starting distance $r_{0}$. In the unbounded system it increases monotonically and approaches an asymptotic value of $\frac{1}{3}$, corresponding to a uniform distribution. We also show results for various degrees of confinement $b$. For $b<3.21 a$ the variance increases monotonically, reaching a maximum value for $r_{0}=b$. For $b>3.21 a$ a maximum occurs at $r_{0}<b$. As $b \rightarrow \infty$ the position of the maximum approaches $r_{0}=2 a$. This should be compared with the $2 \mathrm{D}$ system for which the variance always increases monotonically (see [58]). In addition, this maximum becomes more pronounced as the confinement eases $(b \rightarrow \infty)$ and may exceed that of the uniform distribution, $\frac{1}{3}$. For the same starting position $r_{0}$, the variance in the confined system is always larger than in the unbounded one if $b>2.93 a$. For $b$ less than this value there is a crossover and for strong confinement the variance maximum is suppressed for all values of $r_{0}<b$ (see Fig. 4).

In summary, the qualitative difference between $2 \mathrm{D}$ and $3 \mathrm{D}$ random searches is consequential for the hitting angle distributions. Our principal results are embodied in Eqs. (5) and (11), which describe the effect of confinement on the hitting angle distribution in two and three dimensions, respectively. In the former, the distribution in the presence of a reflecting barrier approaches that of the unbounded system rapidly with increasing wall distance. In three dimensions the bounded distribution also converges quickly as the degree of confinement is reduced, but the limit differs from the unbounded system by a constant additive factor. It may be possible to obtain the full hitting distribution in two dimensions for an arbitrary confining boundary using a recently introduced conformal mapping approach [52]. We have focused on the physically most interesting dimensions, but one could generalize to an arbitrary dimension [36].

Returning to the scenarios presented in the introduction, the animal searching for water in a closed territory always suffers from the presence of the boundary which indirectly pushes it to the front, dangerous part, of the lake. The effect is reversed for a diffusive therapeutic molecule in a cell if the molecular gateway is farther than $\sim 2.8 a$ from the targeted nucleus of radius $a$. In this case, the boundary differentially favors arrival opposite the entry point.

Acknowledgments. We thank Olivier Bénichou and Sid Redner for helpful discussions.
[1] A. Okubo and S. A. Levin, Diffusion and Ecological Problems: Modern Perspectives (Springer, New York, 2001).

[2] J. R. Potts and M. A. Lewis, Proc. R. Soc. B 281, 20140231 (2014).

[3] D. Bearup and S. Petrovskii, J. Theor. Biol. 367, 230 (2015).

[4] S. Gal, S. Alpern, and J. Casas, J. R. Soc. Interface 12, 20150861 (2015).

[5] J. Davis, K. Pitt, A. Olds, A. Harborne, and R. Connolly, Mar. Ecol. Prog. Ser 581, 135 (2017).

[6] S. Mohapatra and P. S. Mahapatra, Sci. Rep. 9, 11258 (2019).

[7] P. C. Bressloff and J. M. Newby, Rev. Mod. Phys. 85, 135 (2013).

[8] E. I. Ozay, G. Gonzalez-Perez, J. A. Torres, J. Vijayaraghavan, R. Lawlor, H. L. Sherman, D. T. Garrigan, A. S. Burnside, B. A. Osborne, G. N. Tew, and L. M. Minter, Mol. Ther. 24, 2118 (2016).
[9] S. Miersch and S. S. Sidhu, F1000Research 5, 1947 (2016).

[10] I. Trenevska, D. Li, and A. H. Banham, Front. Immunol. 8, 1001 (2017).

[11] Y. Lanoiselée, N. Moutal, and D. S. Grebenkov, Nat. Commun. 9, 4398 (2018).

[12] S. Redner, A Guide to First-Passage Processes (Cambridge University Press, Cambridge, 2001).

[13] R. Metzler, G. Oshanin, and S. Redner, First-Passage Phenomena and Their Applications (World Scientific, Singapore, 2014).

[14] M. F. Shlesinger, J. Phys. A: Math. Theor. 42, 434001 (2009).

[15] S. Ro and Y. W. Kim, Phys. Rev. E 96, 012143 (2017).

[16] T. Chou and M. R. D’Orsogna, in First-Passage Phenomena and Their Applications, edited by R. Metzler, G. Oshanin, and S. Redner (World Scientific, Singapore, 2014), p. 306.

[17] A. Gabel, S. N. Majumdar, N. K. Panduranga, and S. Redner, J. Stat. Mech. (2012) P05011. 
[18] G. M. Viswanathan, M. G. Da Luz, E. P. Raposo, and H. E. Stanley, The Physics of Foraging: An Introduction to Random Searches and Biological Encounters (Cambridge University Press, Cambridge, 2011).

[19] F. Bartumeus, D. Campos, W. S. Ryu, R. Lloret-Cabot, V. Méndez, and J. Catalan, Ecol. Lett. 19, 1299 (2016).

[20] C. L. Faustino, L. R. da Silva, M. G. E. da Luz, E. P. Raposo, and G. M. Viswanathan, Europhys. Lett. 77, 30002 (2007).

[21] V. Kurella, J. C. Tzou, D. Coombs, and M. J. Ward, Bull. Math. Biol. 77, 83 (2014).

[22] N. Mizumoto and S. Dobata, Sci. Rep. 8, 3356 (2018).

[23] A. T. Vanak, M. Thaker, and R. Slotow, Biol. Conserv. 143, 2631 (2010).

[24] S. Bell and E. M. Terentjev, Sci. Rep. 7, 17272 (2017).

[25] K. Qiu, K.-f. Gao, L.-j. Yang, Z.-k. Zhang, R. Wang, H.-s. Ma, and Y. Jia, Sci. Rep. 7, 9890 (2017).

[26] L. Mirny, M. Slutsky, Z. Wunderlich, A. Tafvizi, J. Leith, and A. Kosmrlj, J. Phys. A: Math. Theor. 42, 434013 (2009).

[27] Y. Zhang and O. K. Dudko, Ann. Rev. Biophys. 45, 117 (2016).

[28] P. Kar, A. G. Cherstvy, and R. Metzler, Phys. Chem. Chem. Phys. 20, 7931 (2018).

[29] E. Barkai and D. A. Kessler, in First-Passage Phenomena and Their Applications (Ref. [16]), p. 502.

[30] M. S. Titcombe and M. J. Ward, SIAM J. Appl. Math. 60, 1767 (2000).

[31] R. Yvinec, M. R. D’Orsogna, and T. Chou, J. Chem. Phys. 137, 244107 (2012).

[32] T. G. Mattos, C. Mejia-Monasterio, R. Metzler, and G. Oshanin, Phys. Rev. E 86, 031143 (2012).

[33] S. Condamin, O. Bénichou, and M. Moreau, Phys. Rev. E 75, 021111 (2007).

[34] O. Bénichou and R. Voituriez, Phys. Rep. 539, 225 (2014).

[35] T. Guérin, N. Levernier, O. Bénichou, and R. Voituriez, Nature (London) 534, 356 (2016).

[36] E. Ben-Naim and P. Krapivsky, J. Phys. A: Math. Theor. 43, 495007 (2010).
[37] A. Godec and R. Metzler, Sci. Rep. 6, 20349 (2016).

[38] D. S. Grebenkov, Phys. Rev. E 81, 021128 (2010).

[39] Z. Schuss, A. Singer, and D. Holcman, Proc. Natl. Acad. Sci. USA 104, 16098 (2007).

[40] D. Holcman and Z. Schuss, SIAM Rev. 56, 213 (2014).

[41] L. Rajendran, H.-J. Knölker, and K. Simons, Nat. Rev. Drug Discov. 9, 29 (2010).

[42] C. W. Hong and Q. Zeng, FEBS Lett. 588, 350 (2013).

[43] N. J. Yang and M. J. Hinner, in Site-Specific Protein Labeling, edited by A. Gautier and M. J. Hinner (Springer, New York, 2014), p. 29.

[44] L. Szewczak, Cell 165, 759 (2016).

[45] J. B. Garnett and D. E. Marshall, Harmonic Measure (Cambridge University Press, Cambridge, 2005), Vol. 2.

[46] D. A. Adams, L. M. Sander, and R. M. Ziff, Phys. Rev. Lett. 101, 144102 (2008).

[47] B. Duplantier, Phys. Rev. Lett. 82, 3940 (1999).

[48] D. S. Grebenkov, Phys. Rev. E 91, 052108 (2015).

[49] B. B. Mandelbrot and C. J. Evertsz, Nature (London) 348, 143 (1990).

[50] J. G. Wendel, Ann. Probab. 8, 164 (1980).

[51] D. S. Grebenkov, Phys. Rev. Lett. 117, 260201 (2016).

[52] D. S. Grebenkov, R. Metzler, and G. Oshanin, New J. Phys. 19, 103025 (2017).

[53] D. S. Grebenkov and J.-F. Rupprecht, J. Chem. Phys. 146, 084106 (2017).

[54] S. A. Isaacson and J. Newby, Phys. Rev. E 88, 012820 (2013).

[55] J. C. Tzou and T. Kolokolnikov, Multiscale Model. Sim. 13, 231 (2015).

[56] O. Bénichou and R. Voituriez, Phys. Rev. Lett. 100, 168105 (2008).

[57] J. Yang, I. Kupka, Z. Schuss, and D. Holcman, J. Math. Biol. 73, 423 (2016).

[58] See Supplemental Material at http://link.aps.org/supplemental/ 10.1103/PhysRevResearch.2.012019 for detailed calculations. 